\title{
Consumption of refined carbohydrate by patients with Crohn's disease in Tel-Aviv-Yafo
}

\author{
K. SilKofF \\ M.B., B.Ch. \\ L. YEGENA* \\ B.Sc. \\ J. F. MAYBERRY \\ B.Sc., M.B.
}

\author{
A. HALlaK* \\ M.D. \\ P. ROZEN* \\ M.D. \\ J. RHODES \\ M.D., F.R.C.P.
}

\author{
R. G. NEWCOMBE \\ Ph.D., F.S.S.
} The Department of Gastroenterology, Ichilov Hospital, *Tel-Aviv-Yafo, University Hospital of Wales,
Cardiff, and Department of Medical Statistics, Welsh National School of Medicine, Cardiff

\begin{abstract}
Summary
In 27 patients with Crohn's disease living in Israel the current intake of refined carbohydrate (total sugars) and added sugar is significantly greater than in healthy controls. The dietary survey was retrospective and showed that patients with Crohn's disease ate $269 \mathrm{~g}$ of monosaccharides and disaccharides each day compared with $192 \mathrm{~g}$ by matched controls. Similar differences were also found at the onset of the symptoms, $314 \mathrm{~g}$ compared with $207 \mathrm{~g}$. These findings were independent of the country of origin or cultural grouping. Several factors are discussed which suggest a secondary rather than causal relationship between sugar consumption and Crohn's disease.
\end{abstract}

\section{Introduction}

Recent studies in Tel-Aviv (Rozen et al., 1979) have shown that the incidence of Crohn's disease is $1 \cdot 28 / 10^{5} /$ year and the prevalence $12 \cdot 3 / 10^{5}$. In contrast, the current figures for Cardiff (Mayberry, Rhodes and Hughes, 1979) are $4 \cdot 8 / 10^{5} /$ year and $55 \cdot 7 / 10^{5}$. The possible role of diet in Crohn's disease has aroused considerable interest because several European studies (Martini and Brandes, 1976; Miller et al., 1976; Kasper et al., 1977; Mayberry, Rhodes and Newcombe, 1978; Thornton, Emmett and Heaton, 1979) have shown a difference in the consumption of carbohydrate, particularly sugar. Because of this, the authors examined some aspects of diet in patients living in Tel-Aviv and compared them with healthy controls from the same nationg and cultural groups.

\section{Method}

The consumption of refined carbohydrate, including added sugar, was assessed in 27 of 50 을 patients with Crohn's disease living in Israel and $\stackrel{\mathscr{Q}}{\overparen{D}}$ treated at Ichilov Hospital, Tel-Aviv-Yafo.

Patients were matched with healthy controls from $\overrightarrow{\overrightarrow{0}}$ the orthopaedic clinic for age, sex and country of 3 origin. Ten of the patients were born in Israel (native) while the rest were immigrants, chiefly from Eastern Europe (6 from Poland, 2 Russian and one 하 Rumanian). Nineteen of the patients were of 3 . Ashkenazi origin (mainly from Eastern Europe and North America) while the other 8 were Sephardic (mainly from the Mediterranean and Middle East). Care was taken to match each patient with a control born in the same country and belonging to the same 음 cultural group of either Ashkenazim or Sephardim. $>$ In those cases where the patient was an immigrant, the control had come to Israel from the same country $N$ during the same year.

Patients were interviewed by a dietitian and medi- $\stackrel{\sim}{\mathcal{N}}$ cal registrar using a structured questionnaire. The $\underset{\omega}{\omega}$ questionnaire was printed in Hebrew and included $\mathcal{F}$ details of age, sex, country of birth and date of $\varphi$ diagnosis. All subjects were asked each question $\overparen{D}$ and their answers were recorded by the medical $\stackrel{\infty}{+}$ registrar who was aware which subjects were $\frac{T}{0}$ 
patients and which were controls. A section of each questionnaire was devoted to the daily intake of certain foods which included fruit, preserves, cakes, biscuits, sweets, chocolate, milk, ice cream and beverages. The quantity eaten during the previous week was estimated by each patient and control and their answer recorded for each item. Sugar added to drinks and cereals was recorded as a separate item. Patients were also asked similar questions about their dietary habits at the onset of symptoms, while each matched control was questioned about diet at the time corresponding with the onset of symptoms in matched patients. The monosaccharide and disaccharide content of the foods (total sugars) was analysed using tables developed by Guggenheim, Kaufman and Reshef (1977) and the daily intake of total sugars and added sugar were analysed by both a Wilcoxon Matched Pairs Signed rank test and,

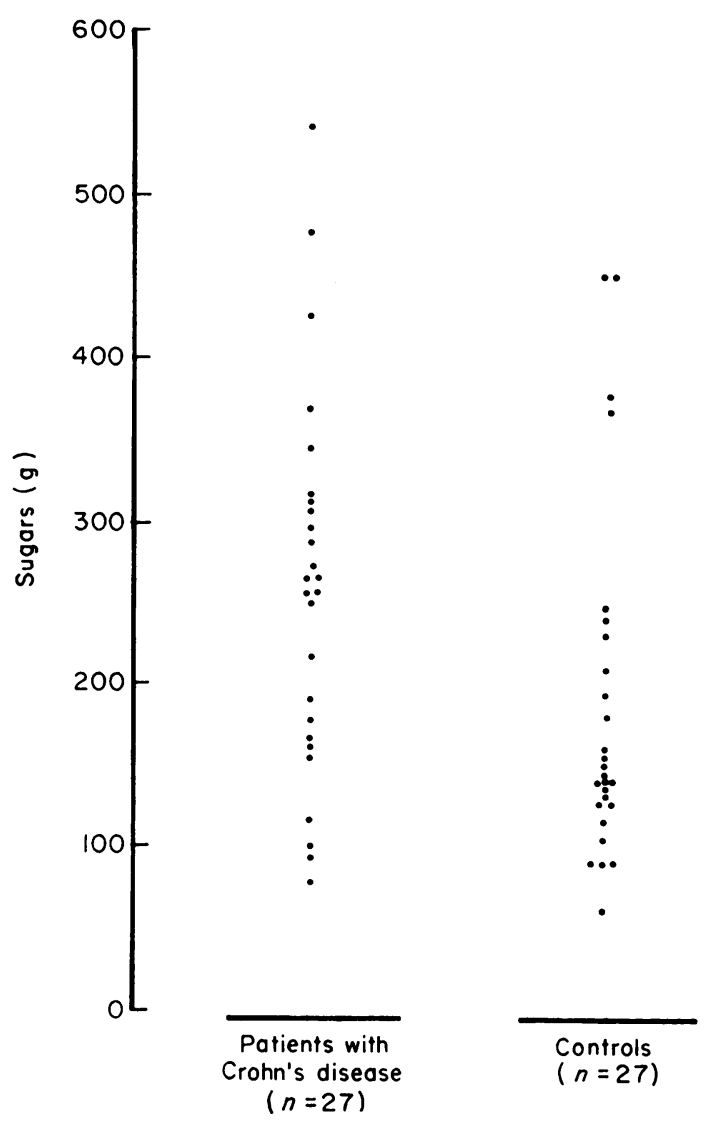

Fig. 1. Current daily intake of monosaccharides and disaccharides in 27 Israeli patients with Crohn's disease and 27 healthy controls matched for age, sex, country of origin and duration of residence in Israel. The intake was assessed by interview and analysis of dietary constituents was made from the tables of Guggenheim et al. (1977). in order to quantify the result, a matched paired $t$ test.

\section{Results}

Patients with Crohn's disease ate an average of $269 \mathrm{~g}$ of total sugars each day compared with $192 \mathrm{~g}$ by matched controls $(z=-2.366, P<0.02 ; t=2.479$, $P=0.02$ ) (Fig. 1). They also ate more added sugar than controls- $35 \mathrm{~g}$ compared with $16 \mathrm{~g}(z=-2 \cdot 222$, $P<0.03 ; t=2.564, P<0.02$ ) (Fig. 2). These differences were less obvious at the onset of the illness but

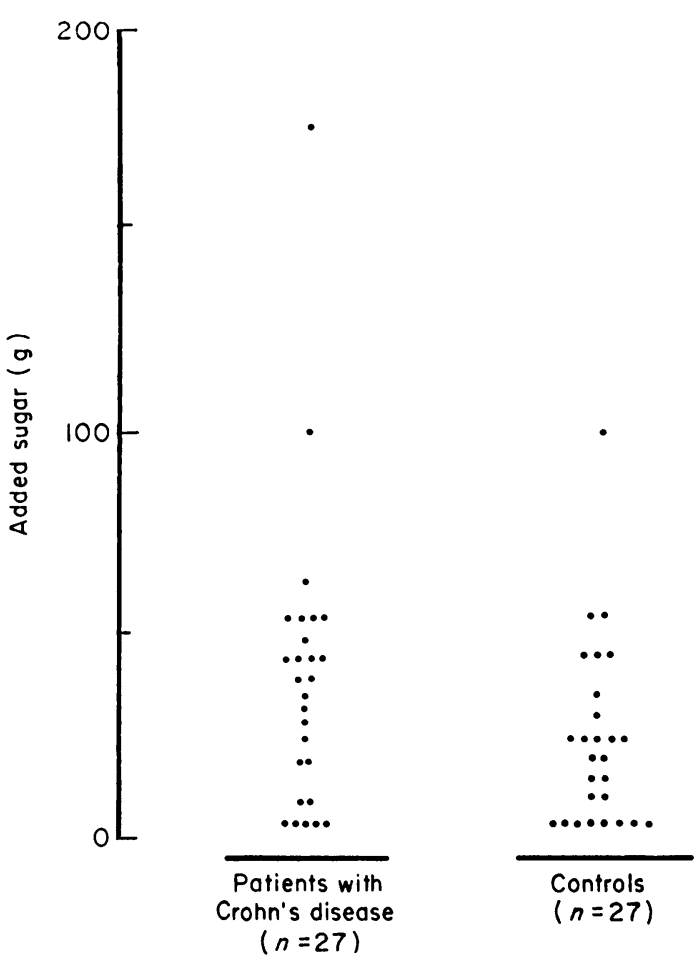

Fig. 2. Current daily intake of sucrose added to beverages and cereals in 27 Israeli patients with Crohn's disease and 28 healthy controls matched for age, sex, country of origin and duration of residence in Israel. The intake was assessed by interview.

nevertheless significant (Table 1). All 27 controls said that there had been no change in their use of sugar and sugar-containing foods, whereas 4 patients had reduced their intake and 2 had increased it during the period under review. The majority of patients (21) felt that their sugar consumption had remained unchanged. This does not agree with the difference between current and past intakes recorded for the patient group in Table 1. 
TABLE 1. Mean sugar consumption ( \pm s.d.) in patients and in controls

\begin{tabular}{|c|c|c|c|c|c|c|}
\hline \multirow[b]{3}{*}{ Mean age $(n=27)$} & \multirow{2}{*}{\multicolumn{2}{|c|}{$\begin{array}{l}\text { Patients with } \\
\text { Crohn's disease }\end{array}$}} & \multirow{2}{*}{\multicolumn{2}{|c|}{ Healthy controls }} & \multicolumn{2}{|c|}{ Tests of significance } \\
\hline & & & & & $\begin{array}{l}\text { Matched paired } \\
t \text { test }\end{array}$ & $\begin{array}{l}\text { Wilcoxon matched } \\
\text { pairs signed } \\
\text { rank test }\end{array}$ \\
\hline & 45 & (17) & 45 & (19) & & \\
\hline Age of males $(n=14)$ & 43 & (18) & 45 & (19) & & \\
\hline Age of females $(n=13)$ & 46 & (16) & 46 & (18) & & \\
\hline Mean duration of symptoms (years) & 8 & (12) & & & & \\
\hline \multicolumn{7}{|l|}{ Current consumption (g) } \\
\hline Total sugars & 269 & $(122)$ & 192 & (113) & $P=0.02$ & $P<0.02$ \\
\hline Added sugar & 35 & (36) & 16 & (16) & $P<0.02$ & $0.05<P<0.1$ \\
\hline \multicolumn{7}{|l|}{ Consumption (g) at onset } \\
\hline Total sugars & 314 & $(164)$ & 207 & (169) & $P<0.05$ & $P<0.03$ \\
\hline Added sugar & 19 & (18) & 16 & (16) & & \\
\hline
\end{tabular}

The intake of monosaccharides and disaccharides is recorded as 'total sugars' and added sugar refers to sucrose added to drinks and cereals.

TABLE 2. Mean refined carbohydrate consumption ( \pm s.d.) in patients with Crohn's disease and in controls

\begin{tabular}{|c|c|c|c|c|c|c|}
\hline \multirow[b]{3}{*}{ Mean age } & \multicolumn{4}{|c|}{$\begin{array}{l}\text { Patients with } \\
\text { Crohn's disease }\end{array}$} & \multicolumn{2}{|l|}{$\begin{array}{l}\text { Matched paired } \\
t \text { test (significance) }\end{array}$} \\
\hline & \multicolumn{4}{|c|}{ Imigrants to Israel $(n=17)$} & & \\
\hline & 51 & (18) & 53 & (19) & & \\
\hline Time in Israel (years) & 29 & (13) & 30 & (12) & & \\
\hline Duration of symptoms (years) & 12 & (14) & & & & \\
\hline \multicolumn{6}{|l|}{ Current consumption (g) } & \\
\hline Total sugars & 270 & (124) & 153 & (71) & $P<0.005$ & 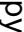 \\
\hline Added sugar & 29 & $(26)$ & 14 & (14) & $0.05<P<0.1$ & छे \\
\hline \multicolumn{7}{|c|}{ Consumption (g) at onset of symptoms } \\
\hline Total sugars & 310 & $(124)$ & 157 & (72) & $P<0.005$ & \\
\hline \multirow[t]{2}{*}{ Added sugar } & 19 & $(16)$ & 11 & (12) & & \\
\hline & \multicolumn{4}{|c|}{ Native Israelis $(n=10)$} & & \\
\hline Mean age & 34 & (10) & 33 & (11) & & \\
\hline Duration of symptoms (years) & 3 & (2) & & & & \\
\hline \multicolumn{6}{|l|}{ Current consumption (g) } & \\
\hline Total years & 267 & $(126)$ & 258 & $(141)$ & & \\
\hline Added sugar & 46 & $(48)$ & 21 & (20) & & \\
\hline \multicolumn{6}{|c|}{ Consumption (g) at onset of symptoms } & \\
\hline Total sugars & 321 & $(152)$ & 291 & $(247)$ & & \\
\hline Added sugar & 19 & (21) & 25 & (19) & & \\
\hline
\end{tabular}

Seventeen of the patients were immigrants to Israel (6 from Poland) and the other 10 were born in Israel. The results of an analysis using a Wilcoxon Matched Pairs Sigred Rank test were similar to those of the matched paired $t$ test.

The current consumption of 'total sugars' and added sugar as well as total sugars intake at the onset of symptoms were all significantly higher in the immigrants with Crohn's disease than in the immigrant controls (Table 2). Both patients and controls appeared to reduce their total sugars and added sugar intake with ageing. The differences observed in native Israeli groups did not reach significance but this may be owing to the smaller size of the groups.

The 19 Ashkenazi patients had a significantly in- creased intake of added sugar currently compared $\supset$ with their matched controls (Table 3), but these differences were not significant in the Sephardim, 을 which was a very small group.

Added sugar is not correlated with total sugars $\stackrel{N}{\circ}$ intake. An analysis considering all possible combin- $\underset{\omega}{ }$ ations of cases and controls, based on 54 subjects and 38 degrees of freedom gave a correlation of 0 +0.229 which was not significant.

It is important to notice the general interest in $\stackrel{\mathscr{C}}{+}$ health and diet taken by patients compared with 
TABLE 3. Mean refined carbohydrate consumption ( \pm s.d.) in patients with Crohn's disease and in controls

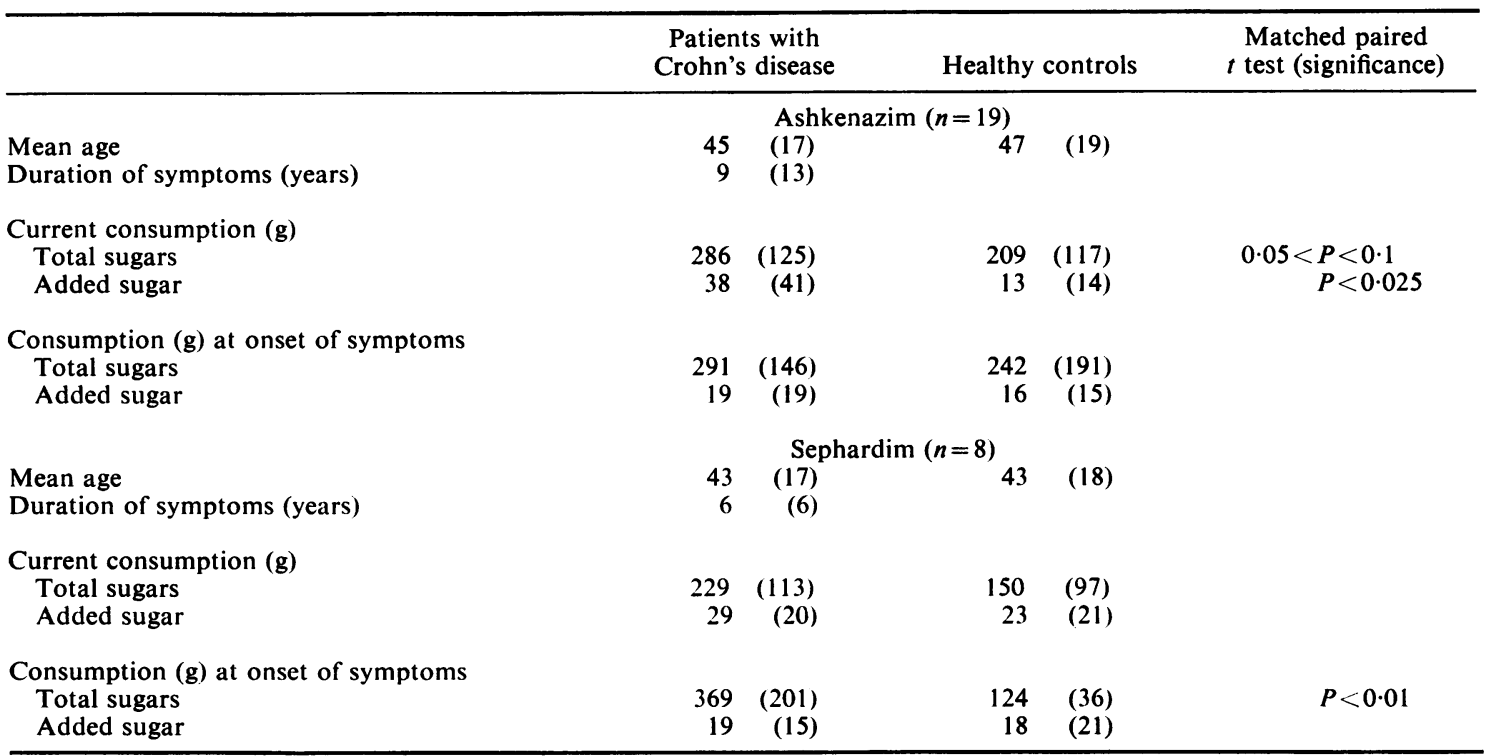

Nineteen patients were of Ashkenazic origin and the other 8 were Sephardim. The results of an analysis using a Wilcoxon Matched Pairs Signed Rank test were similar to those of the matched paired $t$ test.

controls. Eighteen patients knew that they had been breast fed as children compared with only 9 controls.

\section{Discussion}

Early in 1979 Rozen et al. drew attention to the differences in the consumption of carbohydrate and fibre in the population of Tel-Aviv and suggested that their relevance to the aetiology of Crohn's disease should be assessed. In the present study of 27 Israeli patients the current consumption of total sugars (refined carbohydrate) is significantly increased. This is true of immigrants to Israel and Askenazim. The difference is not significant for patients born in Israel or Sephardim which probably reflects the small size of the group. Although less significant, a difference in sugar consumption was present when symptoms began.
Sixty-five per cent. of the population of Tel-AvivYafo belong to the Ashkenazi community (Statistical Year Book for 1978); 70\% of the patients in this study also belonged to this group. The remainder of the patients were designated Sephardic and Oriental. Crohn's disease has a low prevalence in Tel-AvivYafo compared with Cardiff. It is consequently of interest to note the differences in sugar consumption between the 2 countries (Table 4). The mean daily consumption of refined beet and cane sugar in Israel is $134 \mathrm{~g}$ compared with $106 \mathrm{~g}$ in Great Britain. The consumption of refined carbohydrates by controls in Tel-Aviv was considerably higher than in Cardiff but the mean intakes were obtained in different ways. The Cardiff study was based on a detailed prospective study of all dietary items over 5 days, whereas the Tel-Aviv-Yafo study was retrospective and is there-

TABLE 4. Refined carbohydrate consumption in patients from Tel-Aviv-Yafo and Cardiff

\begin{tabular}{lcccc}
\hline & $\begin{array}{c}\text { Prevalence of } \\
\text { Crohn's disease } \\
\text { (cases/10 } \text { population) }\end{array}$ & Patients & $\begin{array}{c}\text { Mean daily intake } \\
\text { (in g) of refined } \\
\text { carbohydrates } \\
\text { controls }\end{array}$ & National studies \\
\hline Tel-Aviv-Yafo & $12 \cdot 3$ & 269 & 192 & 134 \\
Cardiff & $55 \cdot 7$ & 112 & 103 & 106 \\
\hline
\end{tabular}

The prevalence of Crohn's disease in Tel-Aviv-Yafo and Cardiff was measured in independent studies. The refined carbohydrate intake of patients and controls in Cardiff was measured in a prospective study of 16 patients. The figures for consumption of refined beet and cane sugar were published by the International Sugar Organisation. 
fore open to criticism, although differences in diet do exist between different groups in Israel (Bavly, 1972; Mayberry et al., 1981). If the cause of Crohn's disease were directly related to sugar consumption one would expect the prevalence to be higher in Israel than it is in Wales, for Israelis eat much more refined carbohydrate than do British people (Sugar Year Book, 1978). Although refined sugar consumption has remained constant in Great Britain throughout this century the incidence of Crohn's disease has increased significantly, suggesting that the relationship between Crohn's disease and high sugar intake is not a direct one. However, recent work by Heaton, Thornton and Emmett (1979) suggests that reducing sugar and increasing fibre intake may improve the course of the illness in Crohn's disease. In contrast, work by McClain, Soutor and Zieve (1980) has shown that many patients with Crohn's disease have an impaired ability to taste sweetness owing to a zinc deficiency secondary to the disease process and it is possible that this accounts for the increase in sugar consumption.

Several independent studies have demonstrated a difference in sugar consumption between patients with Crohn's disease and controls. It is probable that this difference is present from early in the disease and is not due to chronic ill health (Mayberry, Rhodes and Newcombe, 1980). The data in this paper suggest that the differences do not depend on culture, and similar findings are now available from West Germany (Martini and Brandes, 1976; Miller et al., 1976; Kasper et al., 1977), the United Kingdom (Mayberry et al., 1978; Thornton et al., 1979) and Israel. However, because of the limitations discussed above, it is felt that the association between consumption of refined carbohydrate and Crohn's disease is unlikely to be more than a secondary association.

\section{References}

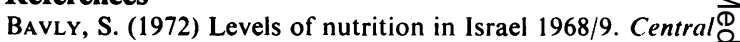
Bureau of Statistics, Special Services No. 368, Jerusalem.C Guggenheim, Y. K., Kaufman, N. A. \& Reshef, A. (1977) Food Composition Tables, 1977. Department of Education and Culture, Government Printing Office, Jerusalem.

Heaton, K. W., Thornton, J. R. \& Emmett, P. M. (1979)을 Treatment of Crohn's disease with an unrefined-carbohydrate, fibre-rich diet. British Medical Journal, 2, 764.

Kasper, H., Rabast, U., SOMmer, H. \& EHL, M. (1977) Untersuchungen zur Hohe des Rohfaserverz ehrs bei 응 Gesunden und bei Erkrankungen des Gastrointestinal-ळ

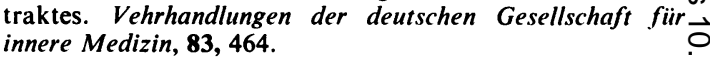

McClain, C., Soutor, C. \& Zieve, L. (1980) Zinc deficiency. $\overrightarrow{\mid}$ A complication of Crohn's disease. Gastroenterology, $\vec{\omega}$ 78, 272.

MARTINI, G. A. \& Brandes, J. W. (1976) Increased consump tion of refined carbohydrates in patients with Crohn's disease. Klinische Wochenschrift, 54, 367

MAYBERRY, J. F., RHODES, J. \& HugheS, L. E. (1979) Incidence of Crohn's disease in Cardiff between 1934 and 1977. S Gut, 20, 602.

MayberRy, J. F., Rhodes, J. \& Newcombe, R. G. (1978) Breakfast and dietary aspects of Crohn's disease. British $\stackrel{\oplus}{\sim}$ Medical Journal, 2, 1401.

MAyberRy, J. F., Rhodes, J. \& Newcombe, R. G. (1980)을 Increased sugar consumption in Crohn's disease. Digestion, 20, 323.

Mayberry, J. F., Rhodes, J., Allan, R., Newcombe, R. G., $\mathbb{D}$ Regan, G. M., Chamberlain, L. M. \& Wragg, K. $\frac{\mathbb{D}}{?}$ (1981) Diet in Crohn's disease; two studies of current and previous habits in newly diagnosed patients. Digestiye믐 Diseases (in press).

Miller, B., Fervers, F., Rohbeck, R. \& Strohmeyer, $\overrightarrow{\mathbf{\beta}}$ (1976) Zuckerhonsum bei Patienten mit Morbus Crohî. Vehrhandlungen der deutschen Gesellschaft für inne Medizin, 83, 922.

Rozen, P., Zonis, J., Yekutiel, P. \& Gilat, T. (1979) Crohn's disease in the Jewish population of Tel-Aviv-Yafo. Gastroenterology, 76, 25.

Statistical Year Book for 1978. Department of Research and $\frac{0}{\mathbb{Q}}$ Statistics, Tel-Aviv-Yafo Municipality.

Sugar Year Book 1978. International Sugar Organization, $\overrightarrow{\vec{B}}$ London.

Thornton, J. R., Emmett, P. M. \& Heaton, K. W. (1979) Diet and Crohn's disease: characteristics of the pre-illness diet. British Medical Journal, $2,762$. 